\title{
Lability of nanoparticulate metal complexes in electrochemical speciation analysis
}

\author{
Herman P. van Leeuwen ${ }^{1} \cdot$ Raewyn M. Town ${ }^{2}$
}

Received: 19 April 2016/Revised: 7 August 2016/Accepted: 19 August 2016 /Published online: 2 September 2016

(C) The Author(s) 2016. This article is published with open access at Springerlink.com

\begin{abstract}
Lability concepts are elaborated for metal complexes with soft (3D) and hard (2D) aqueous nanoparticles. In the presence of a non-equilibrium sensor, e.g. a voltammetric electrode, the notion of lability for nanoparticulate metal complexes, M-NP, reflects the ability of the M-NP to maintain equilibrium with the reduced concentration of the electroactive free $\mathrm{M}^{2+}$ in its diffusion layer. Since the metal ion binding sites are confined to the NP body, the conventional reaction layer in the form of a layer adjacent to the electrode surface is immaterial. Instead an intraparticulate reaction zone may develop at the particle/ medium interface. Thus the chemodynamic features of $\mathrm{M}$ NP complexes should be fundamentally different from those of molecular systems in which the reaction layer is a property of the homogeneous solution $\left(\mu=\left(D_{\mathrm{M}} / k_{\mathrm{a}}^{\prime}\right)^{1 / 2}\right)$. For molecular complexes, the characteristic timescale of the electrochemical technique is crucial in the lability towards the electrode surface. In contrast, for nanoparticulate complexes it is the dynamics of the exchange of the electroactive metal ion with the surrounding medium that governs the effective lability towards the electrode surface.
\end{abstract}

Keywords Intraparticulate reaction layer - Chemodynamics · Ion exchange $\cdot$ Lability $\cdot$ Metal complexes

On the occasion of the retirement of M. Lovrić and S. Komorsky-Lovrić

Herman P. van Leeuwen

herman.vanleeuwen@wur.nl

1 Physical Chemistry and Soft Matter, Wageningen University \& Research, Stippeneng 4, 6708 WE Wageningen, The Netherlands

2 Department of Physics, Chemistry and Pharmacy, University of Southern Denmark, Campusvej 55, 5230 Odense, Denmark

\section{Introduction}

Aqueous nanoparticles (NPs) generally carry electric charge and may have unique ionic reactivity which can even outweigh the reactivity of the molecular counterparts $[1,2]$. Their physicochemical features are a consequence of the reactive sites being spatially confined to the particle body (3D case) or the particle surface (2D), either of them being at a potential different from that in the bulk medium. Accordingly, NPs are finding wide application in e.g. medicine and consumer goods [3]. The diverse range of NPs includes those of natural origin, e.g. humic substances and proteins, and various types of engineered NPs, e.g. $\mathrm{TiO}_{2}, \mathrm{Ag}$, and nanoplastics. The nature of such NPs encompasses hard (impermeable), core-shell, and soft (permeable) ones. All of these types of NPs are ubiquitous in environmental and biological media where their reactivities impact on the chemical speciation of trace inorganics, e.g. metal ions, as well as organic molecules. Thus there is an urgent need to rigorously understand the chemodynamic features of nanoparticulate complexants in order to predict their environmental impact as well as to facilitate tailoring of their reactivities.

Non-equilibrium speciation techniques, such as various electrochemical methods [4], diffusive gradients in thin film (DGT) [5], and dynamic solid phase microextraction (SPME) [6], provide a measure of the fluxes and reactivities of species [7-9]. The pertaining knowledge is operationally formulated in terms of the lability of the complex species, i.e. the extent to which complexes dissociate to release free electroactive species on the timescale of their diffusion towards the sensor surface, e.g. an electrode or a binding phase $[5,10]$. Electrochemical techniques are particularly attractive for 
these studies because a wide range of operational timescales is accessible [11], and other processes such as possible adsorption of NP-bound electroactive species onto the reactive interface can be probed [12]. In the case of dispersed particles incorporating electroactive components, the voltammetric behaviour of individual particles has been observed via the current peaks that arise upon their contact with the electrode surface [13, 14]. Here we explore the nature of kinetic currents of nanoparticulate metal complexes with dissociative limitations in the release of the electroactive species, i.e. the free $\mathrm{M}^{2+}$.

Lability is involved with the dissociative contribution of the complex non-reactive species to the analytical signal of the target reactive species, e.g. the free metal ion or a free organic molecule. Conventional criteria for lability are based upon the implicit assumption that the dimensions of the complexing ligand are on the molecular scale and hence always smaller than the thickness of the nonequilibrium reaction layer that extends over the sensing interface. In such cases, the approximative KouteckýKoryta (KK) scheme [15-17] has been remarkably helpful in solving a wide variety of lability issues [17-22]. It describes the transition from complexation equilibrium control (with coupled diffusion of the complex species) to kinetic control (with dissociation rate limitation) at the reaction layer boundary. However, for nanoparticulate complexing agents this KK scheme will require reconsideration because the reactive sites are not randomly distributed over the medium but confined to the particle body. For a nanoparticulate complex, the nature of lability seems to be rather concerned with local exchange dynamics of the free metal ion at the particle/medium interface [23]. The purpose of the present study therefore is to reconsider the effective lability features for the case of nanoparticulate metal complexes. In doing so, we shall focus on the cases of soft (3D) and hard (2D) aqueous nanoparticulate metal complexes and elaborate the theoretical concepts that define their effective lability in electrochemical metal speciation.

\section{Dynamics of metal-NP complexation}

The Eigen mechanism for aqueous metal ion complexation by simple molecular ligands [24] has been elaborated to describe the overall process of metal ion binding by $3 \mathrm{D}$ and $2 \mathrm{D} \mathrm{NP}$ complexants $[25,26]$. The mechanism distinguishes several steps, any one of which may be rate-limiting for the overall association or dissociation reaction as illustrated for the $3 \mathrm{D}$ case in Fig. 1.

It is evident from Fig. 1 that the electrostatic field of a charged NP can have a significant effect on the operational

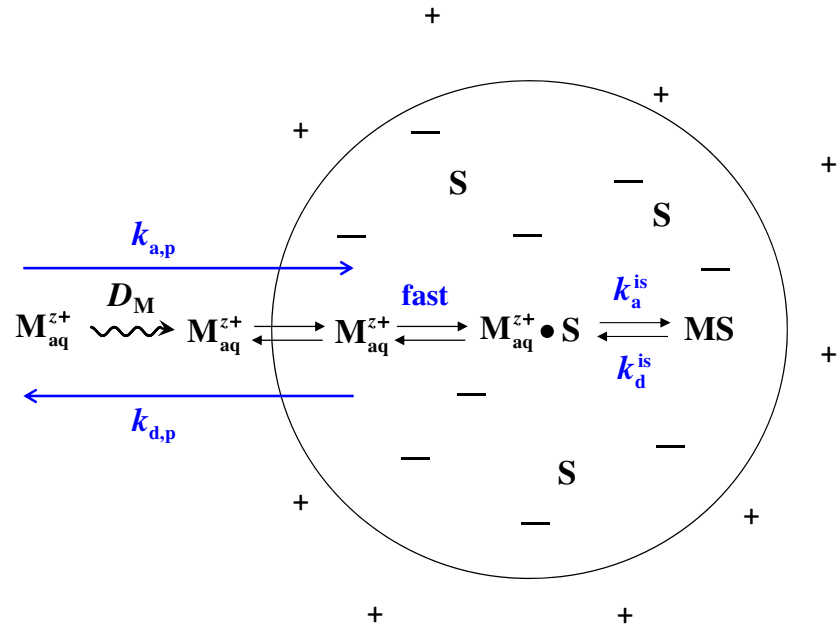

Fig. 1 Schematic representation of the Eigen mechanism for stepwise complexation of a hydrated metal ion, $\mathrm{M}_{\mathrm{aq}}^{z+}$, with a nanoparticulate complexant containing charged or uncharged binding sites (S) and charged sites $(-)$. The + 's denote the extraparticulate counterionic atmosphere. The rate constant for diffusive supply, $k_{\mathrm{a}, \mathrm{p}}$, applies to diffusion of $\mathrm{M}_{\mathrm{aq}}^{z+}$ from the bulk solution to the surface of the complexant and its crossing of the solution/particle interface to be incorporated within the particle as a free hydrated ion. The rate constant for inner-sphere complex formation, $k_{\mathrm{a}}^{\text {is }}$, includes the loss of water of hydration by $\mathrm{M}_{\mathrm{aq}}^{z+}$ and formation of a chemical bond with site $\mathrm{S}$. In the high charge density regime, there is rapid interconversion between $\mathrm{M}_{\mathrm{aq}}^{z+}$ and the outer-sphere associate $\mathrm{M}_{\mathrm{aq}}^{z+} \cdot \mathrm{S}$ (see text). The dissociative counterparts for the rate constants are denoted by, $k_{\mathrm{d}, \mathrm{p}}$ and $k_{\mathrm{d}}^{\text {is }}$ respectively. Adapted from [27]

magnitude of the various rate constants. Indeed, the association rate of a soft charged NP with an oppositely charged ionic reactant can be orders of magnitude greater than that of its molecular counterparts $[1,2]$. This property is a consequence of the effect of the nanoparticulate electrostatic field on (i) the local concentrations of ionic reactants species within the NP body, at partition equilibrium governed by a Boltzmann accumulation factor, $\bar{f}_{\mathrm{B}}$, and (ii) the conductive acceleration of the diffusive supply of ions with opposite charge sign, described by a factor $\bar{f}_{\text {el,a }}$. The effects hold for all types of charged NPs, but are most dramatic for soft NPs in which the accumulation is involved with the volume of the NP (3D). The details of the pertaining processes have been elaborated in previous work $[1,2,7,25]$ and are briefly recalled below. For convenience, unless otherwise stated, the expressions refer to the high charge density regime for the case of $\kappa_{\mathrm{p}} r_{\mathrm{p}}>1$, where $\kappa_{\mathrm{p}}^{-1}$ is the intraparticulate Debye length [28]. Since lability at a macroscopic reactive interface is concerned with the relative rates of metal complex dissociation and macroscopic diffusive supply towards the interface, we first formulate the rate expressions in terms of concentrations averaged over the entire dispersion. 


\section{Complex formation}

For the case of diffusive supply of $\mathrm{M}^{z+}$ towards a spherical charged NP body, the limiting rate, $R_{\mathrm{a}, \mathrm{p}}$ per site $\mathrm{S}$ can be generally written as [27]:

$R_{\mathrm{a}, \mathrm{p}}=4 \pi N_{\mathrm{Av}} r_{\mathrm{p}} D_{\mathrm{M}} \bar{f}_{\mathrm{el}, \mathrm{a}} c_{\mathrm{M}}^{*} c_{\mathrm{S}}^{*} / N_{\mathrm{S}}\left[\mathrm{mol} \mathrm{m} \mathrm{m}^{-3} \mathrm{~s}^{-1}\right]$

with corresponding rate constant:

$k_{\mathrm{a}, \mathrm{p}}=4 \pi N_{\mathrm{Av}} r_{\mathrm{p}} D_{\mathrm{M}} \bar{f}_{\mathrm{el}, \mathrm{a}} / N_{\mathrm{S}} \quad\left[\begin{array}{lll}\mathrm{m}^{3} & \mathrm{~mol}^{-1} & \mathrm{~s}^{-1}\end{array}\right]$

where $c_{\mathrm{S}}^{*}$ is the smeared-out concentration of reactive sites per unit volume of solution, and $N_{\mathrm{S}}$ is the number of reactive sites per particle; $c_{\mathrm{p}}$ is the concentration of particles in the dispersion, equal to $c_{\mathrm{S}}^{*} / N_{\mathrm{S}}$.

The limiting rate of Eigen type inner-sphere complex formation [29], $R_{\mathrm{a}}^{\mathrm{is}}$, for the situation in which the Boltzmann distribution of charged species between medium and particle is at equilibrium, is given by [30]:

$R_{\mathrm{a}}^{\mathrm{is}}=k_{\mathrm{w}} V^{\mathrm{os}} \bar{f}_{\mathrm{B}} N_{\mathrm{Av}} c_{\mathrm{M}}^{*} c_{\mathrm{S}}^{*} \quad\left[\mathrm{~mol} \mathrm{~m} \mathrm{~m}^{-3} \mathrm{~s}^{-1}\right]$

with corresponding rate constant:

$k_{\mathrm{a}}^{\text {is }}=k_{\mathrm{w}} V^{\mathrm{os}} \bar{f}_{\mathrm{B}} N_{\mathrm{Av}} \quad\left[\mathrm{m}^{3} \mathrm{~mol}^{-1} \mathrm{~s}^{-1}\right]$

where $k_{\mathrm{w}}$ is the rate constant of inner-sphere dehydration of the metal ion and $V^{\text {os }}$ is the outer-sphere volume per site $\mathrm{S}$.

As already noted, in the high charge density regime the free metal ion and the outer-sphere associates are electrostatically nearly equivalent and interconversion between these species is very fast [1]. Accordingly the outer-sphere formation step generally is not rate-limiting (Fig. 1).

\section{Complex dissociation}

The limiting rate of diffusion of released $\mathrm{M}^{z+}$ from the spherical particle body into the medium is generally given by

$R_{\mathrm{d}, \mathrm{p}}=\frac{3 D_{\mathrm{M}} \bar{f}_{\mathrm{el}, \mathrm{d}}}{r_{\mathrm{p}}^{2} \bar{f}_{\mathrm{B}}} c_{\mathrm{M}, \mathrm{p}}^{*} \quad\left[\mathrm{~mol} \mathrm{\textrm {m } ^ { - 3 }} \mathrm{s}^{-1}\right]$

where $\bar{f}_{\mathrm{el}, \mathrm{d}}$ is the coefficient for conductive diffusion away from the NP and $c_{\mathrm{M}, \mathrm{p}}^{*}$ is the smeared-out concentration of intraparticulate free metal ion. The corresponding diffusioncontrolled rate constant, $k_{\mathrm{d}, \mathrm{p}}$, is given by:

$$
k_{\mathrm{d}, \mathrm{p}}=3 D_{\mathrm{M}} \bar{f}_{\mathrm{el}, \mathrm{d}}\left(1+K_{\mathrm{int}} c_{\mathrm{S}}^{*}\right) / r_{\mathrm{p}}^{2} \bar{f}_{\mathrm{B}} \quad\left[\mathrm{s}^{-1}\right]
$$

where $K_{\text {int }}$ is the conventional intrinsic stability constant of the inner-sphere complex, usually expressed in terms of smearedout concentrations.

The limiting rate of dissociation of inner-sphere complexes, MS, is given by:

$R_{\mathrm{d}}^{\text {is }}=\frac{k_{\mathrm{a}}^{\text {is }}}{K_{\text {int }} \bar{f}_{\mathrm{B}}} c_{\mathrm{MS}}^{*} \quad\left[\mathrm{~mol} \mathrm{~m}^{-3} \mathrm{~s}^{-1}\right]$

where $k_{\mathrm{a}}^{\text {is }}$ is given by Eq. 4 and $c_{\mathrm{MS}}^{*}$ is the smeared-out concentration of MS. The rate constant for inner-sphere dissociation, $k_{\mathrm{d}}^{\text {is }}$, follows as

$$
k_{\mathrm{d}}^{\text {is }}=k_{\mathrm{a}}^{\text {is }} / K_{\text {int }} \bar{f}_{\mathrm{B}}=k_{\mathrm{w}} V^{\mathrm{os}} N_{\mathrm{Av}} / K_{\text {int }} \quad\left[\mathrm{s}^{-1}\right]
$$

The overall process in which $\mathrm{M}$ is released from the particle body involves consecutively dissociation of inner-sphere MS followed by diffusion of the ensuing $\mathrm{M}^{2+}$ from the particle into the surrounding medium (Fig. 1). Accordingly, the ratelimiting step can be governed by either $k_{\mathrm{d}, \mathrm{p}}$ or $k_{\mathrm{d}}^{\text {is }}$. We note that diffusion inside particles with a substantial water content usually is fast in comparison to extraparticulate diffusion in the aqueous medium [31].

\section{Lability concepts}

Conventional concepts for description of the kinetic features of metal complexes are concerned with the timescales of processes in the bulk volume as well as those at a reactive interface. Bulk volume processes involve the timescale for equilibration of partitioning and complexation processes between the NP and the bulk medium. Processes at a reactive interface, e.g. an electrode, that reduces free $\mathrm{M}^{2+}$ after its release from the NP, involve the dynamic nature of the local association/ dissociation reactions of the M-NP in response to the changing concentration of free $\mathrm{M}$ in the surrounding medium. At the level of a volume complexation reaction in the bulk medium, we distinguish between a complex system that is (i) dynamic, i.e. the timescale of interest is much longer than the characteristic lifetimes of free M and M-NP implying that there is frequent interchange between these two species, and (ii) inert, i.e. a change in species concentrations is not followed by significant re-equilibration.

Accordingly, a system is dynamic at the level of the volume complexation reaction if [17]:

$k_{\mathrm{a}}^{\prime} t, \quad k_{\mathrm{d}} t \quad>>1$

where the applicable $k_{\mathrm{a}}^{\prime}$ and $k_{\mathrm{d}}$ are the composed rate constants for the overall association and dissociation processes, respectively, and $k_{\mathrm{a}}^{\prime}=k_{\mathrm{a}} c_{\mathrm{S}}^{*}$ where $c_{\mathrm{S}}^{*}$ is the smeared-out 
concentration of reactive sites in the bulk dispersion (see previous section).

At the level of an interfacial reaction at an electrode surface, we also need the concept of lability [10], which compares the rate of metal complex dissociation with the rate of diffusive supply towards the interface.

\section{Lability of metal complexes with simple ligands}

We briefly recall the situation for simple ligands with the socalled lability index, $\mathscr{B}$, formulated as the ratio between the dissociation rate controlled flux, $J_{\text {kin }}$, and the diffusive flux towards the reactive interface, $J_{\text {dif }}^{*}$ :

$\mathscr{B}=J_{\text {kin }} / J_{\text {dif }}^{*}$

For $\mathscr{B}>>1$, the system is labile, i.e. equilibrium is maintained on all relevant spatial scales, and the steady-state flux corresponds to the purely diffusion-controlled coupled transport of the free $\mathrm{M}^{2+}$ and the complex ML:

$J_{\mathrm{dif}}^{*}=\bar{D}\left(c_{\mathrm{M}}^{*}+c_{\mathrm{ML}}^{*}\right) / \bar{\delta} \quad\left[\mathrm{mol} \mathrm{m} \mathrm{m}^{-2} \mathrm{~s}^{-1}\right]$

where $\bar{\delta}$ is the mean diffusion layer thickness as determined by $\bar{D}$, the mean diffusion coefficient for the complex system:

$\bar{D}=\frac{D_{\mathrm{M}} c_{\mathrm{M}}+D_{\mathrm{ML}} c_{\mathrm{ML}}}{c_{\mathrm{M}, \mathrm{t}}}=D_{\mathrm{M}}\left(\frac{1+\varepsilon K^{\prime}}{1+K^{\prime}}\right) \quad\left[\begin{array}{ll}\mathrm{m}^{2} & \left.\mathrm{~s}^{-1}\right]\end{array}\right.$

where $\varepsilon=D_{\mathrm{ML}} / D_{\mathrm{M}}$.

At the other limit with $\mathscr{B}<<1$, the system is nonlabile and the metal ion accumulation flux is kinetically controlled, i.e. governed by the rate of dissociation of ML. The ensuing flux is given by:

$J_{\text {kin }}=k_{\mathrm{d}} c_{\mathrm{ML}}^{*} \lambda \quad\left[\mathrm{mol} \mathrm{m} \mathrm{m}^{-2} \mathrm{~s}^{-1}\right]$

where $k_{\mathrm{d}}$ is the rate constant for dissociation of ML, and $\lambda$ denotes the thickness of the reaction layer adjacent to the electrode surface. The reaction layer represents the interfacial zone in which the equilibrium between free $\mathrm{M}$ and the complex ML is distorted [32]. The magnitude of $\lambda$ derives from the mobility of free $\mathrm{M}$ in the aqueous dispersion, i.e. its diffusion coefficient, $D_{\mathrm{M}}$, and its mean free lifetime $1 / k_{\mathrm{a}}^{\prime}$ (where $k_{\mathrm{a}}^{\prime}$ $=k_{\mathrm{a}} c_{\mathrm{L}}^{*}$.), as well as the mobility of the complex $\mathrm{ML}\left(\approx D_{\mathrm{L}}\right)$ and its mean free lifetime $\left(1 / k_{\mathrm{d}}\right)[10,33]$ :

$\lambda=\left(\frac{k_{\mathrm{a}}^{\prime}}{D_{\mathrm{M}}}+\frac{k_{\mathrm{d}}}{D_{\mathrm{L}}}\right)^{-1 / 2} \quad[\mathrm{~m}]$
For very stable complexes with $\varepsilon K^{\prime}>>1$, the general expression for the reaction layer, Eq. (14), reduces to the well-known equation for the (associative) reaction layer, $\mu[33]$ :

$\mu=\left(\frac{k_{\mathrm{a}}^{\prime}}{D_{\mathrm{M}}}\right)^{-1 / 2} \quad[\mathrm{~m}]$

in which $\mu$ is determined by the mobility of free $\mathrm{M}$ and its rate of reassociation with a binding site.

The lability index, $\mathscr{B}$, cf. Eq. (10), follows as

$\mathscr{B}=k_{\mathrm{d}} \mu \bar{\delta} / \bar{D}$

The above expressions are applicable to smaller electrodes, e.g. microelectrodes with radius $r_{0}$, by replacing $\bar{\delta}$ in the expressions by $\left(1 / \bar{\delta}+1 / r_{0}\right)^{-1}$, so long as $r_{0}$ remains greater than $\mu$ (see below).

\section{Lability of nanoparticulate metal complexes}

For convenience we consider the interface to be an electrode at a sufficiently negative potential so that the concentration of free $\mathrm{M}^{2+}$ in the medium side of the electrode/medium interface is zero, i.e. a perfect sink. Furthermore we consider the practically relevant case for which (i) $\varepsilon K^{\prime}>>1$ (sufficiently strong complexes), and (ii) the size of the NP is smaller than the characteristic dimension of the electrode, i.e. $r_{\mathrm{p}}<<\bar{\delta}$ (or $r_{0}$ ).

\section{The soft NP case}

The reaction layer concept for metal complex species $[15,16]$ is based on the rate of reassociation and therefore only has meaning in the presence of complexing ligand sites, S. Indeed, the mere existence of a conventional reaction layer will be confined to local spots along the electrode surface so long as (parts of) particle bodies are present. On the other hand, each individual NP may develop its own reaction layer in the form of an intraparticulate reactive outer shell at the interface between the NP and the medium. This reaction layer may govern the rate of release of free metal ions into the medium. The fundamental notion of the association reaction layer thickness, Eq. (15), still holds, but the applicable $k_{\mathrm{a}}^{\prime}$ for the 3D case now is the intraparticulate one, i.e.

$\mu_{\mathrm{NP}, 3 \mathrm{D}}=\left(\frac{k_{\mathrm{a}}^{\mathrm{is}} c_{\mathrm{S}}}{D_{\mathrm{M}}}\right)^{-1 / 2}[\mathrm{~m}]$ 
where the pertaining $k_{\mathrm{a}}^{\text {is }}$ corresponds to:

$k_{\mathrm{a}}^{\text {is }}=k_{\mathrm{w}}\left(c_{\mathrm{M} \cdot \mathrm{s}} / c_{\mathrm{M}, \mathrm{f}} c_{\mathrm{S}}\right) \quad\left[\mathrm{m}^{3} \mathrm{~mol}^{-1} \mathrm{~s}^{-1}\right]$

where $c_{\mathrm{M}} \cdot \mathrm{S}, c_{\mathrm{M}, \mathrm{f}}$ and $c_{\mathrm{S}}$ are the local intraparticulate concentrations of the outer-sphere complexes, the free metal ion and the reactive sites, respectively. In the high charge density regime $\left(c_{\mathrm{M}} \cdot \mathrm{s} / c_{\mathrm{M}}\right.$, f $)$ is of order unity (see above), thus Eq. (17) reduces to

$\mu_{\mathrm{NP}, 3 \mathrm{D}}=\left(D_{\mathrm{M}} / k_{\mathrm{w}}\right)^{1 / 2} \quad[\mathrm{~m}]$

Accordingly, for rapidly dehydrating metal ions such as $\mathrm{Pb}^{2+}$ and $\mathrm{Cu}^{2+}$ with $k_{\mathrm{w}}$ of the order of $10^{9} \mathrm{~s}^{-1}$ [34], $\mu_{\mathrm{NP}, 3 \mathrm{D}}$ is of the order of $10^{-9} \mathrm{~m}$. This distance is comparable to the dimensions of a free metal ion and the time for diffusion over such distance is the same as that for an infinitely fast chemical reaction without transport limitation. In contrast, for a slowly dehydrating metal ion such as $\mathrm{Ni}^{2+}$, with $k_{\mathrm{w}}=3 \times 10^{4} \mathrm{~s}^{-1}$ [34], $\mu_{\mathrm{NP}, 3 \mathrm{D}}$ is ca. $2 \times 10^{-7} \mathrm{~m}$, i.e. larger than the radius of the NP itself. In this situation the kinetics of dissociation will be an important limiting factor in the rate of release of $\mathrm{Ni}^{2+}$ into the medium.

Since for macroscopic electrodes $\delta>>r_{\mathrm{p}}$, the whole surface of an individual NP experiences essentially the same extent of disequilibration in response to the changing concentration of free $\mathrm{M}^{2+}$ in the steady-state diffusion layer. The outcome is a constant release of $\mathrm{M}^{2+}$ in all directions finally resulting in an increased metal ion flux towards the electrode surface. The extent to which the M-NP can maintain local equilibrium with free $\mathrm{M}^{2+}$ in the steady-state diffusion layer depends on the relative timescales for dissociation of MS within the particle body and diffusion of the ensuing $\mathrm{M}^{2+}$ into the surrounding medium. Intraparticulate diffusion generally is an order of magnitude faster than extraparticulate diffusion [31]. Accordingly, description of the dynamic behaviour of 3D M-NP complexes reduces to comparison of the characteristic timescale for extraparticulate diffu-

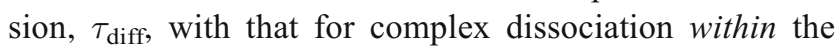
particle body, $\tau_{\text {diss }}$.

The timescale for extraparticulate diffusion is given by:

$\tau_{\text {diff }}=1 / k_{\mathrm{d}, \mathrm{p}} \quad[\mathrm{s}]$

where $k_{\mathrm{d}, \mathrm{p}}$ is given by Eq. (6).

The timescale for complex dissociation within the particle body, $\tau_{\text {diss }}$,

$$
\tau_{\text {diss }}=1 / k_{\mathrm{d}}^{\text {is }} \quad[\mathrm{s}]
$$

The dynamic index for a nanoparticulate complex, thus corresponds to:

$D_{p}=\tau_{\text {diff }} / \tau_{\text {diss }}$

where, on the characteristic timescale of the macroscopic experiment $\bar{\delta} / \bar{D})$, full equilibration of nanoparticulate complexes with the surrounding medium is obtained under conditions of $D_{p}>>1$.

The dynamic index for nanoparticulate complexes is shown as a function of $r_{\mathrm{p}}$ and $k_{\mathrm{w}}$ in Fig. 2 for the 3D case. Two limiting cases can be distinguished, based on the magnitude of $k_{\mathrm{w}}$ :

(i) For a rapidly dehydrating metal ion, e.g. $\mathrm{Pb}\left(\mathrm{H}_{2} \mathrm{O}\right)_{6}^{2+}$, with $k_{\mathrm{w}}$ of the order $10^{10} \mathrm{~s}^{-1}, \mu_{\mathrm{NP}}$ is of the order of $1 \mathrm{~nm}$, i.e. $r_{\mathrm{p}}>>\mu_{\mathrm{NP}}$ and $\not_{p}>>1$. The flux of metal from the NP body towards the electrode surface is then the diffusion controlled one, $J_{\text {diff }}$, given by Eq. (11).

(ii) For a slowly dehydrating metal ion, e.g. $\mathrm{Ni}\left(\mathrm{H}_{2} \mathrm{O}\right){ }_{6}^{2+}$, with $k_{\mathrm{w}}$ of the order of $10^{4} \mathrm{~s}^{-1}, \mu_{\mathrm{NP}}$ is larger than the dimensions of most NPs and $D_{p}<<1$. The entire NP body acts as a reaction layer and the flux of metal from all over its surface towards the medium is the kinetically controlled one, $J_{\text {kin }}$. Since $\tau_{\text {diss }}$ refers to the timescale for dissociation of inner-sphere MS within the particle body, Eq. (21), $J_{\text {kin }}$ is expressed in terms of the local parameters, i.e.

$$
J_{\text {kin }}=k_{\mathrm{d}}^{\text {is }} c_{\mathrm{MS}} V_{\mathrm{p}} / A_{\mathrm{p}} \quad\left[\mathrm{mol} \mathrm{\textrm {m } ^ { - 2 }} \mathrm{s}^{-1}\right]
$$



Fig. 2 Relationship between the metal ion inner-sphere dehydration rate constant, $k_{\mathrm{w}}$, and the dynamic index for 3D nanoparticulate metal complexes for a range of nanoparticle radii, $r_{\mathrm{p}}$. The red dashed line shows the $k_{\mathrm{w}}$ at which $D_{p}=0.02$ and the blue dashed line corresponds to the $k_{\mathrm{w}}$ at which $D_{p}=50$. For illustrative purposes, the binding affinity, reactive site density and charge density are taken as the same for all $r_{\mathrm{p}}$ : computations are performed for $K_{\text {int }}=1 \mathrm{~m}^{3} \mathrm{~mol}^{-1}, c_{\mathrm{S}}=700 \mathrm{~mol} \mathrm{~m}^{-3}$, $\bar{f}_{\mathrm{B}}=5000$ 
where $k_{\mathrm{d}}^{\text {is }}$ derives from the local $k_{\mathrm{a}}^{\text {is }}$ (Eq. (18)) and $K_{\text {int }}, c_{\mathrm{MS}}$ is the intraparticulate concentration of $\mathrm{MS}, V_{\mathrm{p}}$ is the volume of the particle and $A_{\mathrm{p}}$ its surface area.

\section{The hard NP case of surface complexation}

For surface complexation reactions, the geometric confinement of the reactive sites to the particle surface means that it is physically impossible for the local reaction layer to be an intraparticulate one. Accordingly, either step in the overall associative and dissociative processes (Fig. 1) may be ratelimiting. Thus, the limiting step in the association process is obtained by setting the rate of diffusive supply, $R_{\mathrm{ad}, \mathrm{p}}=\left(D_{\mathrm{M}} /\right.$ $\left.r_{\mathrm{p}} N_{\mathrm{S}}\right) c_{\mathrm{M}}^{*}$ against that for inner-sphere complex formation $R_{\mathrm{ad}}^{\text {is }}=k_{\mathrm{ad}}^{\text {is }} c_{\mathrm{M}}^{*} \Gamma_{\mathrm{S}}$, where $\Gamma_{\mathrm{S}}$ is the surface concentration of reactive sites. It has been shown that the Eigen type complexation mechanism also applies to aqueous metal ion binding by oxidic surfaces in aqueous systems [26]. This means that the basic relationship between $k_{\mathrm{a}}^{\text {is }}$ and $k_{\mathrm{w}}$ (Eq. (4)) remains intact for formation of oxidic surface complexes provided that the applicable values for $V^{\mathrm{os}}$ and $f_{\mathrm{B}}$ are inserted. For the regime of large excess of surface binding sites over bound metal ions, the ratio $c_{\mathrm{MS}} / c_{\mathrm{M}}$ is approximately constant. This situation is often formulated in terms of a linear adsorption "isotherm" for which the surface concentration of $\mathrm{M}$ in the form of $\mathrm{MS}, \Gamma_{\mathrm{M}}$, is linearly related to the concentration of free $\mathrm{M}$ in the medium, $c_{\mathrm{M}}^{*}$ :

$\Gamma_{\mathrm{M}}=K_{\mathrm{H}} c_{\mathrm{M}}^{*} \quad\left[\mathrm{~mol} \mathrm{~m}^{-2}\right]$

where $K_{\mathrm{H}}$ is the so-called Henry coefficient. The equilibrium condition requires that adsorption and desorption rates for $c_{\mathrm{M}}^{*}$ and the corresponding $\Gamma_{\mathrm{M}}^{*}$ are equal, implying that the composed rate constants for adsorption and desorption, $k_{\mathrm{ad}}$ and $k_{\mathrm{des}}$, are simply related by $k_{\mathrm{ad}}=k_{\mathrm{des}} K_{\mathrm{H}}$. In the diffusioncontrolled limit we now have only extraparticulate diffusion with $k_{\mathrm{ad}, \mathrm{p}}$ equal to $\left(D_{\mathrm{M}} / r_{\mathrm{p}} N_{\mathrm{S}}\right)$ and the corresponding $k_{\mathrm{des}, \mathrm{p}}$ given by $\left(D_{\mathrm{M}} / r_{\mathrm{p}} K_{\mathrm{H}}\right)$. E.g. for the adsorption process, the limiting diffusion rate is $\left(D_{\mathrm{M}} / r_{\mathrm{p}} N_{\mathrm{S}}\right) c_{\mathrm{M}}^{*}$ which should be set against the limiting rate of formation of MS at the surface, i.e. $k_{\mathrm{ad}}^{\text {is }} c_{\mathrm{M}}^{*} \Gamma_{\mathrm{S}}$ (cf. Eq. (4)). Thus the ratio $\left(D_{\mathrm{M}} / r_{\mathrm{p}} N_{\mathrm{S}}\right) / k_{\mathrm{ad}}^{\text {is }}$ $\Gamma_{\mathrm{S}}$ determines which of the two steps is actually limiting the rate of surface complex formation. In terms of corresponding time constants the ratio comes to $\tau_{\text {ad }} / \tau_{\text {diff }}$ with the dissociation/ desorption counterpart following similarly from the ratio $\left(D_{\mathrm{M}} / r_{\mathrm{p}} K_{\mathrm{H}}\right) / k_{\text {des }}^{\text {is }}$. These results are basically similar to the correction coefficient for the overall association and dissociation rate constants $k_{\mathrm{a}}$ and $k_{\mathrm{d}}$ as proposed by Pinheiro et al. [23] and experimentally verified using metal complexes with silica NPs and surface-functionalized latex NPs [35, 36]. As is clear now from the present analysis, the derived "lability" is actually an expression of the local chemodynamics of the particle in the surrounding medium. Note that for both the $3 \mathrm{D}$ and $2 \mathrm{D}$ cases, the chemodynamic features at this level are primarily governed by the particle's rate constants for uptake/release of $\mathrm{M}$ which are bulk parameters of the system.

Whilst the extremes of diffusion control and kinetic control for both soft and hard nanoparticles are clear-cut, the intermediate mixed control situation is rather involved. Even for simple ligands, only numerical solutions are currently available [37]. For the more involved case of soft nanoparticulate complexes the situation becomes further confounded by the relative magnitudes of $\mu_{\mathrm{NP}}$ and $r_{\mathrm{p}}$. It is evident from Fig. 2 that many soft nanoparticulate complexes with aqueous transition metal ions - with the majority of them having a $k_{\mathrm{W}}$ between $10^{7}$ and $10^{9} \mathrm{~s}^{-1}$ - will fall within the region of mixed diffusive and kinetic control.

\section{Conclusions and outlook}

New chemodynamic concepts are developed to qualitatively describe the operational lability of soft and hard nanoparticulate metal complexes. The framework is conceptually different from that for molecular scale metal complexes, for which the applicable reaction layer is that which extends along the entire sensor surface. In the case of nanoparticle dispersions such a macroscopic reaction layer is immaterial because of the inhomogeneous physical distribution of the metal binding sites over the dispersion volume. Instead, it is the local dynamics of exchange of free $\mathrm{M}$ between particle and medium that govern the effective lability of M-NP. For the 3D case, the chemodynamic features of nanoparticulate metal complexes are determined by the relative timescales for dissociation of inner-sphere complexes within the particle body and the ensuing extraparticulate diffusion of the resulting free $\mathrm{M}^{2+}$ into the surrounding medium. Accordingly, a local reaction layer inside the particle body adjacent to its interface with the medium is a key element in the interpretation. As the size of the metal complexes decreases from the nanoparticulate to the molecular scale, the dynamic criteria presented herein will transition to the conventional molecular ones. The formulation of this transition should be a next step in the development of chemodynamic theory for nanoparticulate complexes.

We note that the analysis herein considers limiting flux conditions at steady-state. During the initial transient stage, in which the macroscopic diffusion layer thickness, grows to its steady-state value, $\delta$, the gradient in the concentration of $\mathrm{M}^{2+}$ in the surrounding medium is changing. For nanoparticulate complexes of transition metal ions with dehydration rate constants in the intermediate range, the uptake/ release rates may not be completely independent of the gradient in $\mathrm{M}^{2+}$ concentration. Accordingly a fully dynamic analysis will be required to properly define establishment of steady-state, or the effective timescale in case of a transient 
technique. In the case of microelectrodes, with characteristic dimensions smaller than the thickness of the macroscopic steady-state solution diffusion layer [38], diffusive transport towards the surface is of a convergent radial nature and thus steady-state is more rapidly established. Commercial microelectrodes typically have dimensions of the order of a few $\mu \mathrm{m}$, i.e. still larger than the NP body and hence also larger than the intraparticulate reaction shell. In such cases the various expressions given in preceding sections remain applicable with $\delta$ being replaced by the characteristic dimension of the microelectrode, e.g. its radius $r_{0}$. Due to the greater magnitude of $J_{\text {diff }}$ the lability of metal complexes with simple ligands is lower at microelectrodes than that at conventional sized ones at which planar diffusion prevails [39-41].

The emergence of nano-sized electrodes in recent years $[40,42-45]$ generates the situation in which the reactive interface is more comparable in size to many NPs. That is, the spatial scale over which there is a concentration gradient in the surrounding medium will be comparable to or smaller than the particle body. Again, this situation will call for a more sophisticated approach in the distribution of release rates over the surface of individual particles at different positions with respect to the nanosensor.

ML, inner-sphere complex with a molecular sized ligand; MS, inner-sphere complex with a nanoparticulate reactive site; $\mathrm{NP}$, nanoparticle; S, reactive site that covalently binds a metal ion; $c_{\mathrm{M}}^{*}$, bulk concentration of free metal ion $\mathrm{M}_{\mathrm{aq}}^{z+}\left(\mathrm{mol} \mathrm{m}^{-3}\right)$; $c_{\mathrm{M}, \mathrm{p}}^{*}$, smeared-out concentration of intraparticulate free metal ion $\mathrm{M}_{\mathrm{aq}}^{z+}\left(\mathrm{mol} \mathrm{m}^{-3}\right) ; c_{\mathrm{M}}$, , intraparticulate concentration of free $\mathrm{M}^{2+}\left(\mathrm{mol} \mathrm{m}{ }^{-3}\right) ; c_{\mathrm{M}} \cdot \mathrm{s}$, intraparticulate concentration of outersphere complexes $\left(\mathrm{mol} \mathrm{m}^{-3}\right) ; c_{\mathrm{MS}}^{*}$, smeared-out concentration of inner-sphere complexes in the bulk dispersion $\left(\mathrm{mol} \mathrm{m}^{-3}\right)$; $c_{\mathrm{MS}}$, intraparticulate concentration of inner-sphere complexes $\left(\mathrm{mol} \mathrm{m}{ }^{-3}\right) ; c_{\mathrm{S}}^{*}$, smeared-out concentration of binding sites in the bulk dispersion $\left(\mathrm{mol} \mathrm{m}^{-3}\right)$; $c_{\mathrm{S}}$, intraparticulate concentration of binding sites $\left(\mathrm{mol} \mathrm{m}^{-3}\right) ; c_{\mathrm{p}}$, concentration of particles in the bulk dispersion $\left(\mathrm{mol} \mathrm{m}^{-3}\right) ; \varepsilon$, ratio of diffusion coefficients, $D_{\mathrm{MS}} / D_{\mathrm{M}} ; \delta$, mean diffusion layer thickness for $\mathrm{M}_{\mathrm{aq}}^{z+}$ and M-NP (m); $D_{\mathrm{M}}$, diffusion coefficient of the metal ion $\mathrm{M}_{\mathrm{aq}}^{z+}$ in aqueous solution $\left(\mathrm{m}^{2} \mathrm{~s}^{-1}\right) ; D$, weighted average of the diffusion coefficients of $\mathrm{M}_{\mathrm{aq}}^{z+}$ and M-NP $\left(\mathrm{m}^{2} \mathrm{~s}^{-1}\right) ; \not_{\rho}$, dynamic index for nanoparticulate complexes; $\bar{f}_{\mathrm{B}}$, Boltzmann equilibrium partitioning factor; $\bar{f}_{\text {el, }}$, electrostatic coefficient for conductive diffusion towards the NP; $\bar{f}_{\text {el. }}$, electrostatic coefficient for conductive diffusion away from the NP; $\Gamma_{\mathrm{M}}^{*}$, equilibrium surface concentration of complexes for a $2 \mathrm{D} \mathrm{NP}\left(\mathrm{mol} \mathrm{m}^{-2}\right) ; \Gamma_{\mathrm{S}}$, surface concentration of reactive sites for a $2 \mathrm{D} \mathrm{NP}\left(\mathrm{mol} \mathrm{m}^{-2}\right) ; J_{\text {dif }}$, diffusion controlled flux $\left(\mathrm{mol} \mathrm{m} \mathrm{m}^{-2} \mathrm{~s}^{-1}\right) ; J_{\text {kin }}$, kinetically controlled flux $\left(\mathrm{mol} \mathrm{m}^{-2} \mathrm{~s}^{-1}\right)$; $\kappa_{\mathrm{p}}^{-1}$, intraparticulate Debye length $(\mathrm{m}) ; K_{\mathrm{H}}$, Henry adsorption coefficient (m); $K_{\text {int }}$, intrinsic stability constant for MS $\left(\mathrm{m}^{3} \mathrm{~mol}^{-1}\right) ; k_{\mathrm{a}}$, overall rate constant for complex formation $\left(\mathrm{m}^{3} \mathrm{~mol}^{-1} \mathrm{~s}^{-1}\right) ; k_{\mathrm{a}, \mathrm{p}}$, rate constant for diffusive supply of $\mathrm{M}_{\mathrm{aq}}^{z+}$ to a $3 \mathrm{D} \mathrm{NP}\left(\mathrm{m}^{3} \mathrm{~mol}^{-1} \mathrm{~s}^{-1}\right) ; k_{\mathrm{a}}^{\text {is }}$, rate constant for inner-sphere complex formation from the precursor outer-sphere complex in a 3D NP $\left(\mathrm{m}^{3} \mathrm{~mol}^{-1} \mathrm{~s}^{-1}\right) ; k_{\text {ads,p }}$, rate constant for diffusive supply of $\mathrm{M}_{\mathrm{aq}}^{z+}$ to a $2 \mathrm{D} \mathrm{NP}\left(\mathrm{m}^{3} \mathrm{~mol}^{-1} \mathrm{~s}^{-1}\right) ; k_{\text {ads }}^{\text {is }}$, rate constant for inner-sphere complex formation from the precursor outersphere complex at the surface of a $2 \mathrm{D} \mathrm{NP}\left(\mathrm{m}^{3} \mathrm{~mol}^{-1} \mathrm{~s}^{-1}\right) ; k_{\mathrm{d}, \mathrm{p}}$, rate constant for diffusion of $\mathrm{M}_{\mathrm{aq}}^{z+}$ away from a $3 \mathrm{D} \mathrm{NP}\left(\mathrm{s}^{-1}\right)$; $k_{\mathrm{d}}^{\mathrm{is}}$, rate constant for inner-sphere complex dissociation in a 3D NP $\left(\mathrm{s}^{-1}\right) ; k_{\mathrm{des}, \mathrm{p}}$, rate constant for diffusion of $\mathrm{M}_{\mathrm{aq}}^{z+}$ away from a $2 \mathrm{D} \mathrm{NP}\left(\mathrm{s}^{-1}\right) ; k_{\text {des }}^{\text {is }}$, rate constant for inner-sphere complex dissociation at the surface of a $2 \mathrm{D} \mathrm{NP}\left(\mathrm{s}^{-1}\right) ; k_{\mathrm{w}}$, innersphere dehydration rate constant of hydrated metal ions $\left(\mathrm{s}^{-1}\right)$; $\mathscr{C}$, lability index for molecular size complexes; $\lambda$, thickness of the reaction layer $(\mathrm{m}) ; \mu$, thickness of the association reaction layer (m); $\mu_{\mathrm{NP}, 3 \mathrm{D}}$, thickness of the intraparticulate reaction layer for a $3 \mathrm{D}$ nanoparticle $(\mathrm{m}) ; \mu_{\mathrm{NP}, 2 \mathrm{D}}$, thickness of the reaction layer for a $2 \mathrm{D}$ nanoparticle $(\mathrm{m}) ; N_{\mathrm{S}}$, number of binding sites $\mathrm{S}$ per NP; $r_{0}$, electrode radius (m); $r_{\mathrm{p}}$, particle radius $(\mathrm{m}) ; R_{\mathrm{a}, \mathrm{p}}$, rate of diffusive supply of $\mathrm{M}_{\mathrm{aq}}^{z+}$ towards a $3 \mathrm{D} \mathrm{NP}\left(\mathrm{mol} \mathrm{m}^{-3} \mathrm{~s}^{-1}\right) ; R_{\mathrm{a}}^{\text {is }}$, rate of inner-sphere complex formation in a $3 \mathrm{D}$ NP $\left(\mathrm{mol} \mathrm{m} \mathrm{m}^{-3} \mathrm{~s}^{-1}\right) ; R_{\mathrm{ad}, \mathrm{p}}$, rate of diffusive supply of $\mathrm{M}_{\mathrm{aq}}^{z+}$ towards a $2 \mathrm{D} \mathrm{NP}\left(\mathrm{mol} \mathrm{m}^{-3} \mathrm{~s}^{-1}\right) ; R_{\text {ad }}^{\text {is }}$, rate of inner-sphere complex formation at the surface of a 2D NP $\left(\mathrm{mol} \mathrm{m}^{-3} \mathrm{~s}^{-1}\right) ; R_{\mathrm{d}, \mathrm{p}}$ rate of diffusive efflux of $\mathrm{M}_{\mathrm{aq}}^{z+}$ away from a $3 \mathrm{D} \mathrm{NP}\left(\mathrm{mol} \mathrm{m}^{-3} \mathrm{~s}^{-1}\right) ; R_{\mathrm{d}}^{\text {is }}$, rate of inner-sphere complex dissociation in a 3D NP $\left(\mathrm{mol} \mathrm{m}^{-3} \mathrm{~s}^{-1}\right)$; $R_{\text {des,p }}$, rate of diffusive efflux of $\mathrm{M}_{\mathrm{aq}}^{z+}$ away from a $2 \mathrm{D} \mathrm{NP}$ $\left(\mathrm{mol} \mathrm{m} \mathrm{m}^{-3} \mathrm{~s}^{-1}\right) ; R_{\mathrm{des}}^{\text {is }}$, rate of inner-sphere complex dissociation at the surface of a 2D NP $\left(\mathrm{mol} \mathrm{m}^{-3} \mathrm{~s}^{-1}\right) ; \tau_{\mathrm{ad}}$, characteristic timescale for adsorption on a $2 \mathrm{D} \mathrm{NP}(\mathrm{s}) ; \tau_{\text {diff, }}$ characteristic timescale for extraparticulate diffusion (s); $\tau_{\text {diss }}$, characteristic timescale for the M-NP dissociation (s); $V^{\text {os }}$, outer-sphere volume for an ion pair between $\mathrm{M}_{\mathrm{aq}}^{z+}$ and an individual site $\mathrm{S}\left(\mathrm{m}^{3}\right)$.

Open Access This article is distributed under the terms of the Creative Commons Attribution 4.0 International License (http:// creativecommons.org/licenses/by/4.0/), which permits unrestricted use, distribution, and reproduction in any medium, provided you give appropriate credit to the original author(s) and the source, provide a link to the Creative Commons license, and indicate if changes were made.

\section{References}

1. van Leeuwen HP, Buffle J (2009) Chemodynamics of aquatic metal complexes: from small ligands to colloids. Environ Sci Technol 43: $7175-7183$

2. van Leeuwen HP, Buffle J, Duval JFL, Town RM (2013) Understanding the extraordinary ionic reactivity of aqueous nanoparticles. Langmuir 29:10297-10302 
3. Wise K, Brasuel M (2011) The current state of engineered nanomaterials in consumer goods and waste streams: the need to develop nanoproperty-quantifiable sensors for monitoring engineered nanomaterials. Nanotechnol Sci Appl 4:73-86

4. Buffle J, Tercier-Waeber ML (2000) In situ voltammetry: concepts and practice for trace analysis and speciation. In: Buffle J, Horvai G (eds) In situ monitoring of aquatic systems: chemical analysis and speciation. John Wiley \& Sons, Chichester, pp. 279-405

5. Galceran J, Puy J (2015) Interpretation of diffusion gradients in thin films (DGT) measurements: a systematic approach. Environ Chem 12:112-122

6. Benhabib K, ter Laak TL, van Leeuwen HP (2008) Steady-state diffusion regime in solid-phase microextraction kinetics. Anal Chim Acta 609:113-119

7. van Leeuwen HP (2011) Steady-state DGT fluxes of nanoparticulate metal complexes. Environ Chem 8:525-528

8. Zielińska K, van Leeuwen HP, Thibault S, Town RM (2012) Speciation analysis of aqueous nanoparticulate diclofenac complexes by solid-phase microextraction. Langmuir 28:14672-14680

9. Town RM, van Leeuwen HP (2014) Labilities of nanoparticulate metal complexes in environmental speciation analysis. Environ Chem 11:196-205

10. van Leeuwen HP, Town RM, Buffle J, Cleven RFMJ, Davison W, Puy J, van Riemsdijk WH, Sigg L (2005) Dynamic speciation analysis and bioavailability of metals in aquatic systems. Environ Sci Technol 39:8545-8556

11. Buffle J (1988) Complexation reactions in aquatic systems: an analytical approach. Ellis Horwood, Chichester

12. van Leeuwen HP, Buffle J, Lovrić M (1992) Reactant adsorption in analytical pulse voltammetry: methodology and recommendations. Pure Appl Chem 64:1015-1028

13. Tsekov R, Kovač S, Žutić V (1999) Attachment of oil droplets and cells on dropping mercury electrode. Langmuir 15:5649-5653

14. Ivošević DeNardis N, Žutić V, Svetličić V, Frkanec R (2009) Amperometric adhesion signals of liposomes, cells and droplets. Chem Biochem Eng Q 23:87-92

15. Koutecký J, Koryta J (1961) The general theory of polarographic kinetic currents. Electrochim Acta 3:318-339

16. Koryta J, Dvorak J, Kavan L (1993) Principles of electrochemistry, 2nd edn. Wiley, Chichester

17. van Leeuwen HP, Puy J, Galceran J, Cecília J (2002) Evaluation of the Koutecký-Koryta approximation for voltammetric currents generated by metal complex systems with various labilities. J Electroanal Chem 526:10-18

18. van Leeuwen HP, Town RM (2004) Stripping chronopotentiometry at scanned deposition potential (SSCP). Part 4. The kinetic current regime. J Electroanal Chem 561:67-74

19. Town RM, van Leeuwen HP (2004) Dynamic speciation analysis of heterogeneous metal complexes with natural ligands by stripping chronopotentiometry at scanned deposition potential (SSCP. Aust J Chem 57:983-992

20. van Leeuwen HP, Town RM (2006) Stripping chronopotentiometry at scanned deposition potential (SSCP). Part 7. Kinetic currents for $\mathrm{ML}_{2}$ complexes. J Electroanal Chem 587:148-154

21. Buffle J, Startchev K, Galceran J (2007) Computing steadystate metal flux at microorganism and bioanalogical sensor interfaces in multiligand systems. A reaction layer approximation and its comparison with the rigorous solution. Phys Chem Chem Phys 9:2844-2855

22. van Leeuwen HP, Town RM (2009) Protonation effects on dynamic flux properties of aqueous metal complexes. Collect Czechoslov Chem Commun 74:1543-1557

23. Pinheiro JP, Minor M, van Leeuwen HP (2005) Metal speciation dynamics in colloidal ligand dispersions. Langmuir 21:8635-8642
24. Eigen M (1963) Fast elementary steps in chemical reaction mechanisms. Pure Appl Chem 6:97-115

25. van Leeuwen HP, Town RM, Buffle J (2011) Chemodynamics of soft nanoparticulate metal complexes in aqueous media: basic theory for spherical particles with homogeneous spatial distributions of sites and charges. Langmuir 27:4514-4519

26. van Leeuwen HP (2008) Eigen kinetics in surface complexation of aqueous metal ions. Langmuir 24:11718-11721

27. Town RM, Duval JFL, Buffle J, van Leeuwen HP (2012) Chemodynamics of metal complexation by natural soft colloids: cu(II) binding by humic acid. J Phys Chem A 116:6489-6496

28. Duval JFL (2005) Electrokinetics of soft interfaces. 2. Analysis based on the nonlinear Poisson-Boltzmann equation. Langmuir 21:3247-3258

29. Eigen M (1963) Fast elementary steps in chemical reaction mechanisms. Pure Appl Chem 6:97-116

30. van Leeuwen HP, Buffle J, Town RM (2012) Electric relaxation processes in chemodynamics of aqueous metal complexes: from simple ligands to soft nanoparticulate complexants. Langmuir 28:227-234

31. Crank J (1979) The mathematics of diffusion. Oxford Science Publications, Oxford

32. Heyrovský J, Kuta J (1966) Principles of polarography. Academic Press, New York

33. Zhang Z, Buffle J, van Leeuwen HP (2007) Roles of dynamic metal speciation and membrane permeability in metal flux through lipophilic membranes: general theory and experimental validation with nonlabile complexes. Langmuir 23:5216-5226

34. Morel FMM, Hering JG (1993) Principles and applications of aquatic chemistry. Wiley-Interscience, New York

35. Pinheiro JP, Domingos RF, Minor M, van Leeuwen HP (2006) Metal speciation dynamics in colloidal ligand dispersions. Part 3: lability features of steady-state systems. J Electroanal Chem 596:57-64

36. Goveia D, Pinheiro JP, Milkova V, Rosa AH, van Leeuwen HP (2011) Dynamics and heterogeneity of $\mathrm{Pb}$ (II) binding by $\mathrm{SiO} 2$ nanoparticles in an aqueous dispersion. Langmuir 27:7877-7883

37. de Jong HG, van Leeuwen HP, Holub K (1987) Voltammetry of metal complex systems with different diffusion coefficients of the species involved. Part I. Analytical approaches to the limiting current for the general case including association/dissociation kinetics. J Electroanal Chem 234:1-16

38. Štulík K, Amatore C, Holub K, Mareček V, Kutner W (2000) Microelectrodes. Definitions, characterization, and applications. Pure Appl Chem 72:1483-1492

39. van Leeuwen HP, Town RM (2002) Stripping chronopotentiometry for metal ion speciation analysis at a microelectrode. J Electroanal Chem $523: 16-25$

40. Galceran J, Puy J, Salvador J, Cecília J, van Leeuwen HP (2001) Voltammetric lability of metal complexes at spherical microelectrodes with various radii. J Electroanal Chem 505:85-94

41. Lovrić M, Komorsky-Lovrić S (2014) Influence of electrode radius on apparent lability of complex of amalgam forming ions. Int $\mathrm{J}$ Electrochem Sci 9:5549-5559

42. Zhang B, Galusha J, Shiozawa PG, Wang G, Bergren AJ, Jones RM, White RJ, Ervin EN, Cauley CC, White HS (2007) Bench-top method for fabricating glass-sealed nanodisk electrodes, glass nanopore electrodes, and glass nanopore membranes of controlled size. Anal Chem 79:4778-4787

43. Li Y, Bergman D, Zhang B (2009) Preparation and electrochemical response of 1-3 nm Pt disk electrodes. Anal Chem 81:5496-5502

44. Zhang Y, Xu S, Qian YY, Yang X, Li Y (2015) Preparation, electrochemical responses and sensing application of $\mathrm{Au}$ nanoelectrodes down to $5 \mathrm{~nm}$. RSC Adv 5:77248-77254

45. Danis L, Gateman SM, Snowden ME, Halalay IC, Howe JY, Mauzeroll J (2015) Anodic stripping voltammetry at nanoelectrodes: trapping of $\mathrm{Mn}^{2+}$ by crown ethers. Electrochim Acta 162:169-175 